Full paper

\title{
Cesium-lead based inorganic perovskite quantum-dots as interfacial layer for highly stable perovskite solar cells with exceeding $21 \%$ efficiency
}

\author{
Seckin Akin ${ }^{\mathrm{a}, \mathrm{b}}$, Yemliha Altintas ${ }^{\mathrm{c}}$, Evren Mutlugun ${ }^{\mathrm{d}, \mathrm{e}}$, Savas Sonmezoglu ${ }^{\mathrm{a}, \mathrm{b}, *}$ \\ ${ }^{a}$ Nanotechnology R\&D Laboratory, Karamanoglu Mehmetbey University, Karaman, Turkey \\ ${ }^{\mathrm{b}}$ Department of Metallurgical and Materials Engineering, Karamanoglu Mehmetbey University, Karaman, Turkey \\ ${ }^{\mathrm{c}}$ Department of Materials Science and Nanotechnology Engineering, Abdullah Gul University, Kayseri, Turkey \\ ${ }^{\mathrm{d}}$ Department of Electrical \& Electronics Engineering, Abdullah Gul University, Kayseri, Turkey \\ ${ }^{\mathrm{e}}$ UNAM - Institute of Materials Science and Nanotechnology, Bilkent University, Ankara, Turkey
}

\section{A R T I C L E I N F O}

\section{Keywords:}

CsPbBrxI3-x inorganic perovskite quantum-

dots

Interfacial layer

Stability

Perovskite solar cells

\begin{abstract}
A B S T R A C T
Despite the excellent photovoltaic performances of perovskite solar cells (PSCs), the instability of PSCs under severe environment (e.g. humidity, light-induced, etc.) limits further commercialization of such devices. Therefore, in recent years, research on the long-term stability improvement of PSCs has been actively carried out in perovskite field. To address these issues, we demonstrated the incorporation of ultra-thin interfacial layer of inorganic $\mathrm{CsPbBr}_{1.85} \mathrm{I}_{1.15}$ perovskite quantum-dots (PQDs) that can effectively passivate defects at or near to the perovskite/hole transport material (HTM) interface, significantly suppressing interfacial recombination. This passivation layer increased the open circuit voltage $\left(\mathrm{V}_{\text {oc }}\right)$ of triple-cation perovskite cells by as much as $50 \mathrm{mV}$, with champion cells achieving $\mathrm{V}_{\mathrm{oc}} \sim 1.14 \mathrm{~V}$. As a result, we obtained hysteresis-free cells with the efficiency beyond $21 \%$. More importantly, devices based on such architecture are capable of resisting humidity and lightinduced. Remarkably, the device employing $\mathrm{CsPbr}_{1.85} \mathrm{I}_{1.15}$ demonstrated a superb shelf-stability aganist to humidity under ambient conditions (R.H. $\geq 40 \%$ ), retaining nearly $91 \%$ of initial efficiency after 30 days, while the efficiency of control device rapidly dropped to $45 \%$ from its initial value under the same conditions. Besides benefiting from the high moisture resistivity as well as supressed ion migration, PSCs based on PQDs showed better operational stability (retaining $94 \%$ of their initial performance) than that of the PQDs-free one under continuous light irradiation over $400 \mathrm{~h}$. In addition, a faster PL decay time of 4.66 ns was attained for perovskite/PQDs structure (5.77 ns for only PQDs structure) due to the favorable energy transfer at the interface, indicating a Förster resonance energy transfer (FRET) mechanism. This work indicates that inorganic PQDs are important materials as interlayer in PSCs to supremely enhance the device stability and efficiency.
\end{abstract}

\section{Introduction}

The hybrid organic - inorganic perovskite materials have recently gained substantial attention due to their high absorption coefficient, good charge carrier mobility, long-range charge diffusion lengths, and good solution process ability, which make them uniquely suitable for photovoltaic applications [1-5]. To date, PSCs with methylammonium lead halide have achieved power conversion efficiency (PCE) of $19.3 \%$ [6], and an efficiency exceeding $21 \%$ has recently been obtained by mixture of formamidinium and methylammonium [1]. Despite the tremendous increase in the PCE, their poor long-term device stability related to degradation in particular by moisture in air and light-induced exposure has not been solved yet and this event may become the biggest obstacle on the path of PSCs towards commercial viability [7].
Currently, researchers focused on the eliminating this problem by $i$ ) modifying the perovskite composition by doping with $\mathrm{Br}$ [8] and/or Cs [9] atoms, ii) controlling the perovskite morphology by incorporating quantum-dots into perovskite film [10-12], or iii) passivating the interface between perovskite/hole-transport material (HTM) layers by carbon-based materials [13], polymers [14], and hydrophobic materials [7]. Another strategy is to synthesize new organic or inorganic HTL materials instead of spiro-OMeTAD [15-19]. Interestingly, these approaches provide the device lifespan longer than the case without these materials, but not long enough to ensure long-term stability, as well as occasionally reducing efficiency. It is the fact that main cause of poor stability occurs due to the weakness of the methylammonium lead halide perovskite and presence of additive/dopants originating from HTL layer arising from their inherent vulnerability to environmental

\footnotetext{
* Corresponding author. Nanotechnology R\&D Laboratory, Karamanoglu Mehmetbey University, Karaman, Turkey.

E-mail address: svssonmezoglu@gmail.com (S. Sonmezoglu).
} 
stresses, such as moisture and oxygen, making the device deteriorate quickly even under low humidity conditions. Therefore, interfaces and boundaries (surface and grain) between perovskite/HTM layers play an important role in PSCs, which can not only promote the charge transport and retard the carrier recombination but also tune the film morphology through controlling the perovskite crystal growth. It is a clear necessity that interlayer/passivation materials have to be carefully selected to synchronously reach the highly efficient photovoltaic performance and stability.

In recent years, quantum dots (QDs) have emerged as promising alternative interlayer materials for improving the stability and efficiency in PSCs due to their high photoluminescence (PL) quantum yields and low excitation energies [20]. Very recently, Wang et al. [10], reported an interface engineering method based on the incorporation of $\mathrm{CH}_{3} \mathrm{NH}_{3} \mathrm{PbBr}_{0.9} \mathrm{I}_{2.1}$ QDs between $\mathrm{CH}_{3} \mathrm{NH}_{3} \mathrm{PbI}_{3}$ perovskite and $\mathrm{HTM}$ layers in PSCs to enhance charge transfer and efficiency. However, such QDs suffer from poor stability and can cause the fluorescence quenching with perovskite layer owing to the volatile organic part. Compared to other QDs [20,21], inorganic perovskite QDs are much superior in terms of having similar lattice constant with photo-induced dipole, adaptable band-gap, thermally stable above $100{ }^{\circ} \mathrm{C}$, presence of stable cation (Cs) and anion (Br), easier solution processing, smaller atomic density and flexible electronic properties [10,11,22-27]. Inspired by above-mentioned promises, we attempted to utilize the inorganic perovskite $\mathrm{CsPbBr}{ }_{1.85} \mathrm{I}_{1.15}$ QDs as interlayer at perovskite/HTM interface to enhance both the long-term stability and efficiency of PSCs, stemming from the physical passivation of the organic perovskite, modifying the effective band gap and observed non-radiative energy transfer among PQDs and perovskite layer. To the best of our knowledge, this is the first example of the employing $\mathrm{CsPbBr}_{1.85} \mathrm{I}_{1.15}$ QDs as an interlayer between triple-cation based perovskite and spiro-OMeTAD in PSCs.

Herein, we propose a simple and very effective one-step solution processing approach as implementing an interfacial layer with spincoated inorganic $\mathrm{CsPbBr}_{1.85} \mathrm{I}_{1.15}$ PQDs on triple-cation mixed-halide $\mathrm{Cs}_{0.05}\left(\mathrm{FA}_{0.85} \mathrm{MA}_{0.15}\right)_{0.95} \mathrm{~Pb}\left(\mathrm{I}_{0.85} \mathrm{Br}_{0.15}\right)_{3} \quad\left[\mathrm{FA}=\quad \mathrm{CH}\left(\mathrm{NH}_{2}\right)_{2}{ }^{+}\right.$, $\mathrm{MA}=\mathrm{CH}_{3} \mathrm{NH}_{3}{ }^{+}$] perovskite layer in the normal (n-i-p) configuration to resist moisture with light-induced degradation and suppress charge recombination simultaneously. By this approach, an additional PQDs layer with the enriched $\mathrm{Br}$ composition lead to drastically improved long-term stability against moisture ( $>40 \%$ relative humidity without encapsulation for 30 days at room temperature), remaining to $19.25 \%$, degrading only $9 \%$ of initial efficiency, and enhanced electron/chargetransport kinetics exhibiting a remarkable PCE up to $21.14 \%$ with respect to reference cell (19.51\%). Besides, when the cells are exposed to continuous light irradiation, the PQDs-modified PSCs could retain $94 \%$ of their initial performance after $400 \mathrm{~h}$ of full-sun illumination. These results suggest that this approach is a promising tool for future device design with a view to achieve both highly efficient and long-term stable solar devices.

\section{Results and discussion}

Our cells had a normal n-i-p architecture (glass/FTO/compact-TiO $/$ mesoporous- $\mathrm{TiO}_{2} / \mathrm{Cs}_{0.05}\left(\mathrm{FA}_{0.85} \mathrm{MA}_{0.15}\right)_{0.95} \mathrm{~Pb}\left(\mathrm{I}_{0.85} \mathrm{Br}_{0.15}\right)_{3}$ perovskite/ $\mathrm{CsPBr}_{1.85} \mathrm{I}_{1.15}$ PQDs/spiro-OMeTAD/Au) and detailed fabrication process is given in the Supporting Information. The digital photo of the PQDs in solvent with and without UV-light can be seen in Fig. 1a. As shown in Fig. 1b, quantum yield (QY) of the corresponding sample is $61 \%$. The narrow size distribution of the sample can be also understood from the pronounced first excitonic peaks and the TEM image. High resolution TEM images of the synthesized samples were shown in Fig. 1c and Fig. S1. Crystal structure of the synthesized sample was characterized with XRD analysis and given in Fig. 1d. Sample was prepared by using spin coating on the silicon glass. The most common planes in cubic $\mathrm{CsPbr}_{3}$ perovskite quantum dots (100), (110), (200),
(211) are oriented in $15.18^{\circ}, 21.55^{\circ}, 30.64^{\circ}$, and $37.77^{\circ}$ with respect to two-theta values in the literature [28]. The same planes in cubic $\mathrm{CsPb}_{3}$ QDs were also reported as $14.32^{\circ}, 20.30^{\circ}, 28.87^{\circ}, 35.55^{\circ}$, XRD pattern of the $\mathrm{CsPBr}_{3}$ PQDs slightly shifts to the small angle values with the substitution of the iodide inside the structure of PQDs [24,25]. The observed planes and peaks in our XRD measurements are $14.76^{\circ}(100)$, $20.98^{\circ}$ (110), $29.6^{\circ}$ (200), 33.2 $2^{\circ}(210), 36.68^{\circ}(211)$ and $42.36^{\circ}(220)$ which resemble the XRD peaks in hybrid structures of $\mathrm{CsPbBr}_{1.85} \mathrm{I}_{1.15}$ PQDs.

Valance states and chemical analysis of synthesized PQDs sample were analyzed with X-ray photoelectron spectroscopy (XPS) measurements as shown in Fig. 2. Binding energy of Cs-3d was observed at $724 \mathrm{eV}\left(3 \mathrm{~d}_{5 / 2}\right)$ and $738 \mathrm{eV}\left(3 \mathrm{~d}_{3 / 2}\right)$, binding energy of Pb-4f was detected at $138.3 \mathrm{eV}\left(4 \mathrm{f}_{7 / 2}\right)$ and $143 \mathrm{eV}\left(4 \mathrm{f}_{5 / 2}\right)$. In Fig. 2 , the peaks in I-3d was measured at $618.6 \mathrm{eV}\left(3 \mathrm{~d}_{5 / 2}\right)$ and $630 \mathrm{eV}\left(3 \mathrm{~d}_{5 / 2}\right)$, to show both peaks of the doublet pairs of Br-3d, the spectra is fitted in Fig. S2 with two peaks which were oriented at $68.32 \mathrm{eV}\left(3 \mathrm{~d}_{5 / 2}\right)$ and at $69.49 \mathrm{eV}$ $\left(3 \mathrm{~d}_{3 / 2}\right)$ binding energies. All elements in the composition of Cs-based PQDs sample were detected by XPS analysis and all binding energies were well matched with the literature [29]. Also, elemental analysis made by XPS analysis reveals that the PQDs has $\mathrm{CsPbBr}_{1.85} \mathrm{I}_{1.15}$ stoichiometry. FT-IR analysis of PQDs was carried out in Fig. S3. Characteristic wave numbers of oleic acid and oleylamine were detected in this measurement in agreement with the ligand effect of the PQD.

To explore the PQDs-based interfacial layer, we investigated the crystalline structure of the perovskite film. Fig. 3a shows X-ray diffraction (XRD) patterns of as-prepared and passivated perovskite films obtained fresh and after 7 days of exposure to ambient conditions maintained at relative humidity of $40-50 \%$. The XRD pattern of asprepared film demonstrates that peaks are consistent with triple-cation perovskite [9], and no characteristic impurity peaks are observed; this implies high purity of the absorber products. However, after 7 days of exposure, the non-perovskite $\delta$ and $\beta$ phase peaks appeared at $11.7^{\circ}$ and $12.6^{\circ}$, respectively, confirming that the triple-cation perovskite film decomposed much faster under humidity. Interestingly, after PQDs modified on triple-cation perovskite layer, since the $\beta$ phase peak decreased dramatically, the $\delta$ phase peak has disappeared as clearly shown in aged XRD pattern of PQDs/perovskite layer. We speculate that the PQDs diffuse into grain boundaries and passivate the charge traps at grain surface and boundaries, and thereby, reducing the ion diffusion by blocking these grain boundary channels, as well as strengthens the bonding. This implies that passivation with PQDs offers advantages in terms of better degradation kinetics, considering that decomposition process was greatly retarded. Taking into account fresh XRD pattern of PQDs/perovskite layer, we can see the reflection from (200) at $30.4^{\circ}$, confirming the formation of cubic PQDs as a separate phase on surface of perovskite layer. Furthermore, no peak shift or intensity change, and width broadening appeared after passivating with PQDs, indicating that PQDs do not affect the phase composition and crystal structure of the triple-cation perovskite film. This is clear evidence that the PQDs remained on the surface rather than penetrating into the perovskite lattice. To further investigate the effects of the PQDs interfacial layer on the light harvesting of perovskite, absorption measurements were carried out with no HTM present (Fig. 3b). Besides, we have further analyzed the absorbance properties of perovskite covered with different concentrations of PQDs (Fig. S4a). From both Fig. 3b and S4b, we can say that the shift of the band edge is not observed, because the absorbance remains approximately constant. It can be attributed to the most of the incident visible light is absorbed by the perovskite film considering illumination from the FTO side, so PQDs are difficult excite to elicit fluorescence or absorption. This finding confirmed again that PQDs has been covered on perovskite layer as interlayer rather than penetrating inside. A series of PSCs with varied concentrations of the PQDs, as well as a control device, were fabricated and J-V scans for these devices were investigated to determine the influence of the concentration of PQDs on the performance of PSCs in Fig. S4c. A striking 
(a)

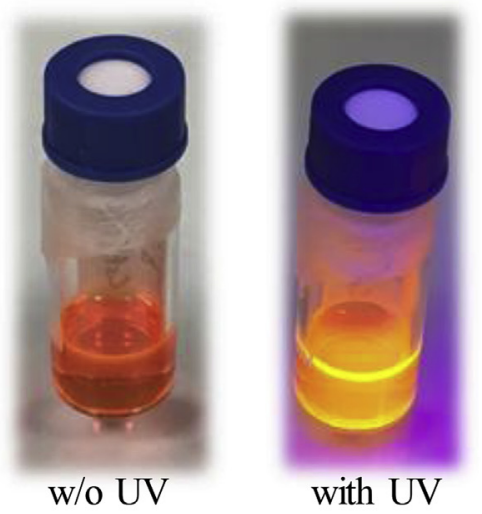

(c)

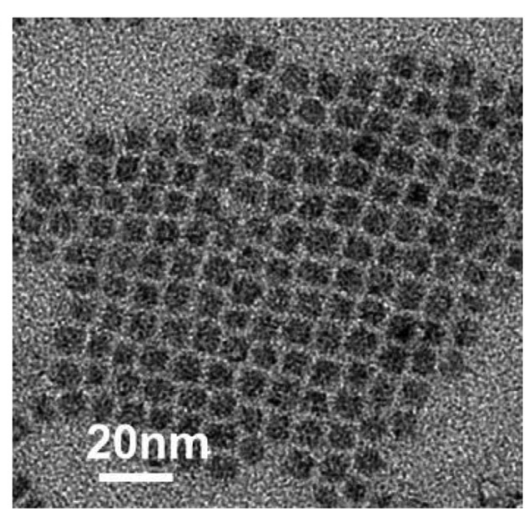

(b)
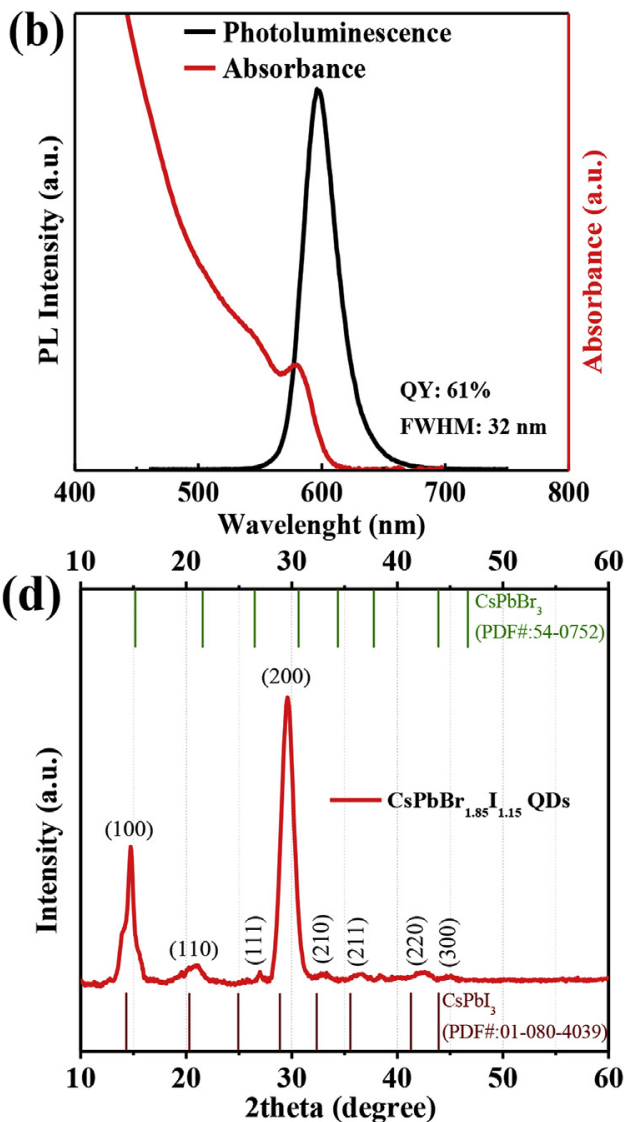

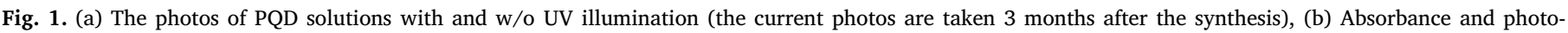
luminescence spectra, (c) high resolution TEM image, (d) XRD spectrum of synthesized sample.
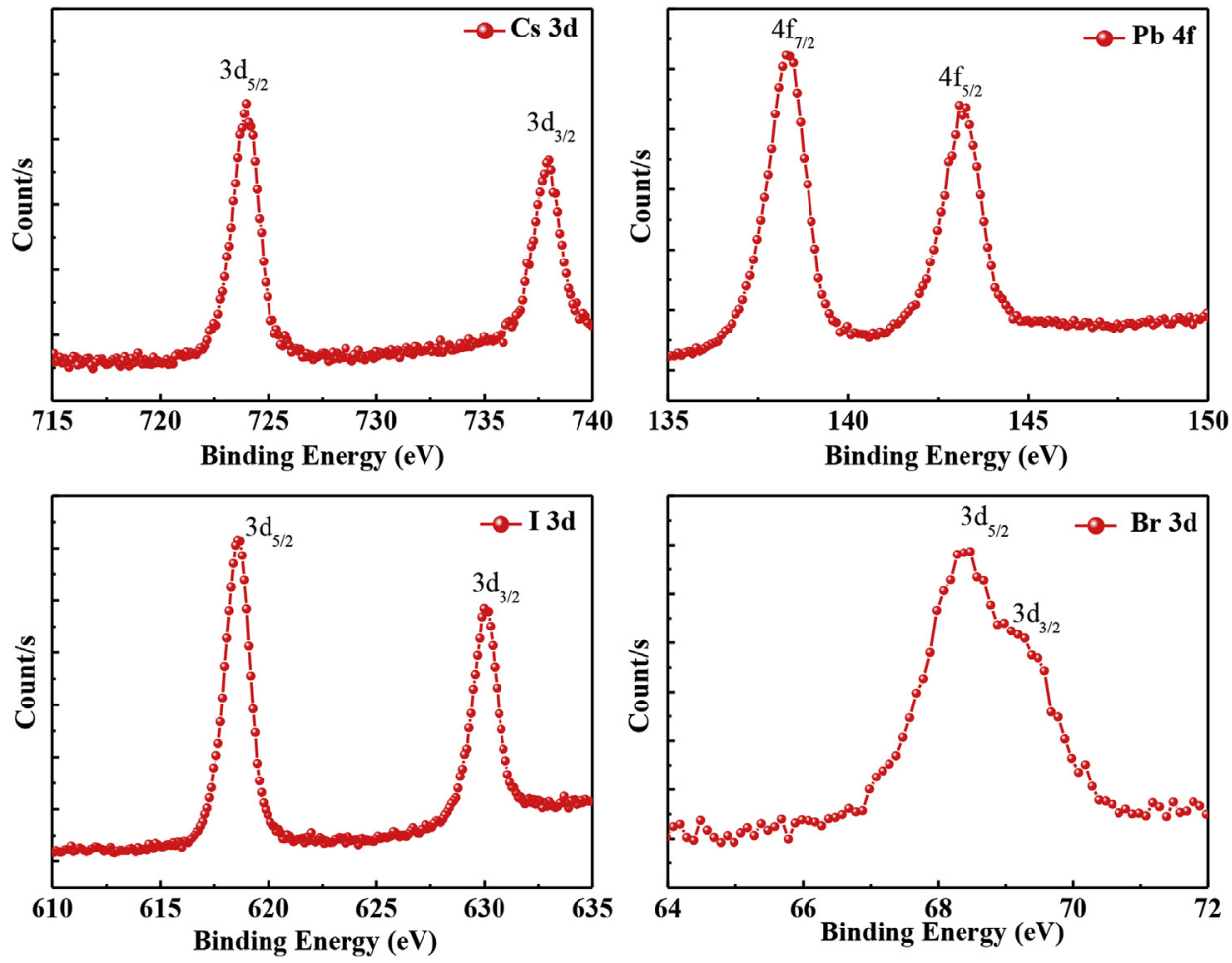

Fig. 2. XPS spectra of inorganic perovskite based QDs. 

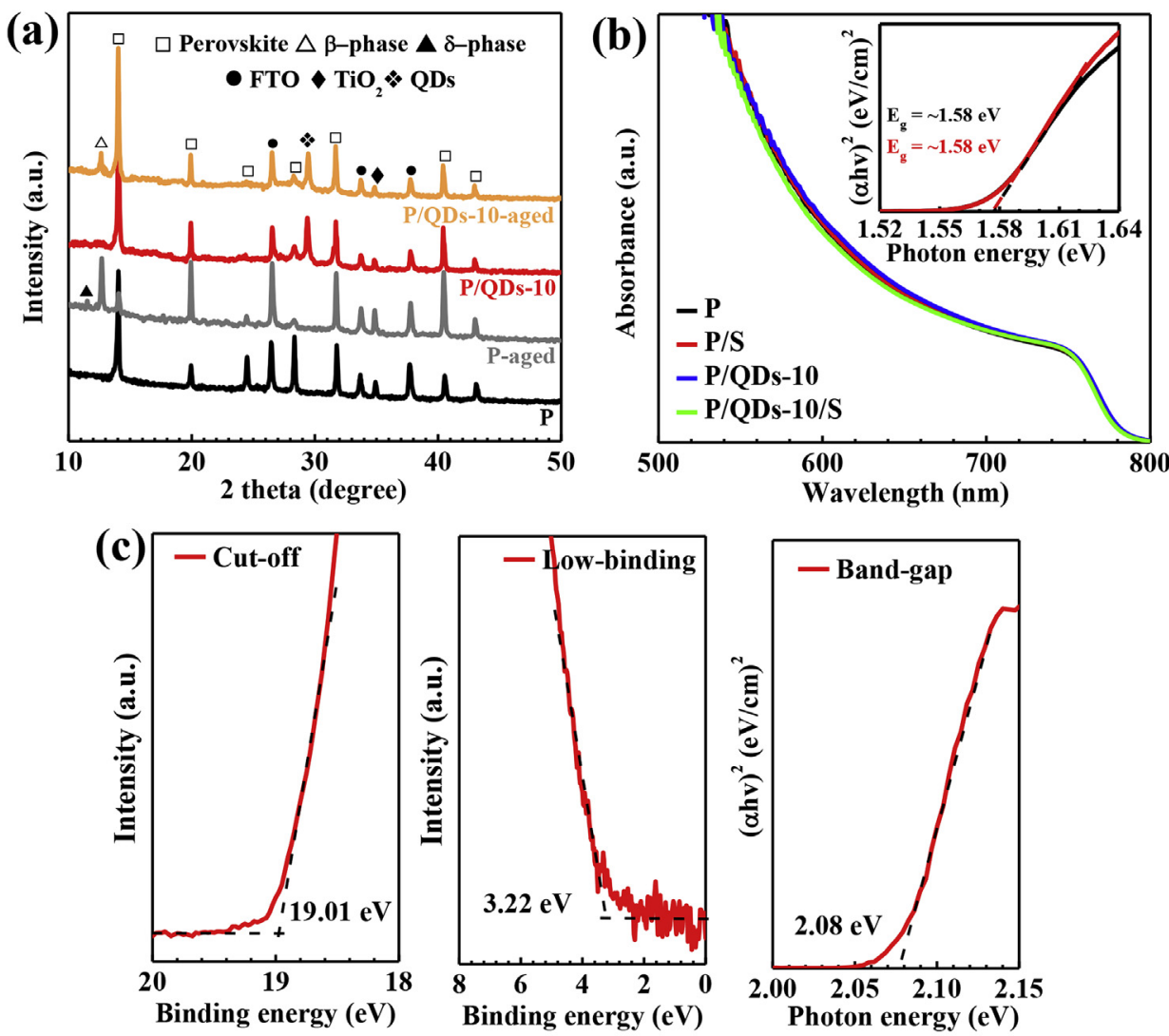

(d)

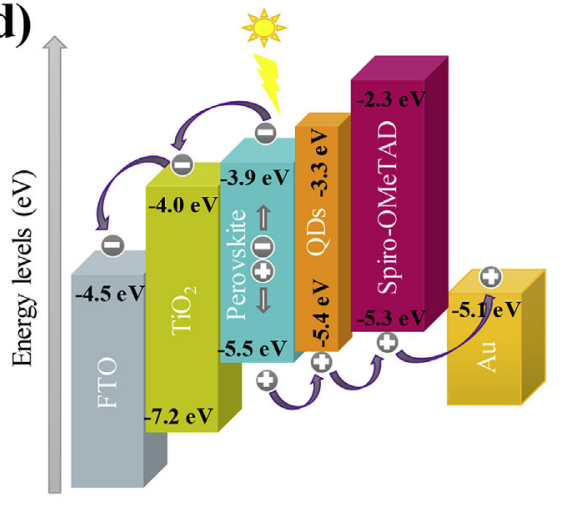

(e)

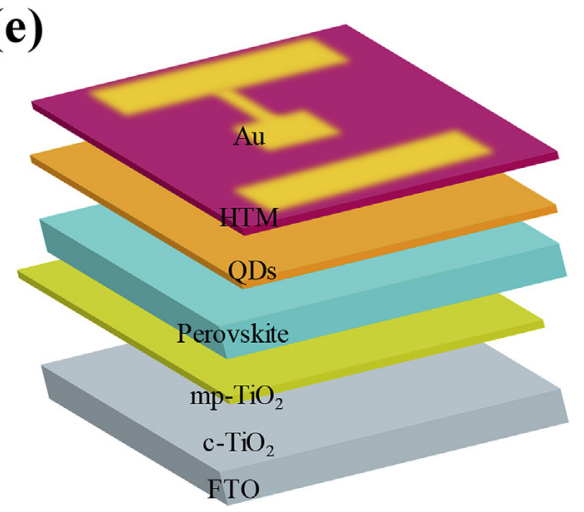

Fig. 3. (a) XRD patterns of the bare and QDs-10 modified perovskite films in fresh and aged forms. (b) UV-vis absorption spectra of the bare and QDs-10 modified perovskite films with and without spiro-OMeTAD layer. The inset shows the Tauc plots of the corresponding films. (c) UPS (cut-off and low binding regions) and bandgap curves of the pure QDs on FTO. The valence band energy $\left(\mathrm{E}_{\mathrm{VB}}\right)$ of the PQDs was determined according to the following equation: $\mathrm{E}_{\mathrm{VB}}=-21.22+\left(\mathrm{E}_{\text {cut-off }}-\mathrm{E}_{\text {onset }}\right)$, where $\mathrm{E}_{\text {onset }}$ is the onset of photoemission in the low binding energy region, $E_{\text {cut-off }}$ is the inelastic cut-off binding energy and $21.22 \mathrm{eV}$ is the energy of the light source $(\mathrm{He} 1 \alpha)$. (d) Schematic representation of energy-level diagram of the materials used in the PSC, with energy levels given in eV. (e) Schematic cross-sectional view of the device architecture. finding is that, compared with the control device, all characteristic parameters of the PQDs-based cells are generally enhanced (Table S1). The best cell conversion efficiency of $20.68 \%$ is achieved with PSC using $10 \mathrm{mg} \mathrm{mL}^{-1}$ PQDs, which is much higher than that of the control device (19.03\%). Further increase of the PQDs concentration to 15 and $20 \mathrm{mg} \mathrm{mL}^{-1}$ leads to gradual decrease of PCE, which is ascribed to higher recombination processes at the interface of perovskite/HTM or unfavorable molecular interaction [30]. Hence, all of the PSCs in our experiments were fabricated under these optimized conditions. To determine the energy levels of PQDs, UV-vis absorption spectroscopy and UV-photoelectron spectroscopy (UPS) were performed. The UV-vis absorption spectra of the PQD solution reveal that the gaps between the conduction band (CB) edge and the valence band (VB) edge, as determined by the wavelength of onset absorption (Fig. 3c), is 2.08. The VB edge, as determined by UPS (Fig. $3 \mathrm{c}$ ), is $-5.41 \mathrm{eV}$ and the CB edge is $-3.33 \mathrm{eV}$, leading to the favored energy level alignment with energy level triple-cation perovskite $[18,31,32]$ and spiro-OMeTAD. The schematic energy level diagram of the perovskite, PQDs, and HTM is presented in Fig. 3d. The wider band gap PQDs on the surface acts as a barrier to reduce charge carrier recombination at the interface between the perovskite and the HTM, leading to higher $\mathrm{V}_{\mathrm{oc}}$, and to prevent backflow of electrons from CB of perovskite to HTM, improving charge collection. Schematic cross-sectional view of the device architecture is given in Fig. 3e.

To investigate the effects of hydrophobic nature of PQDs interlayer on the surfaces of perovskite films, we focused on their water wetting behavior by means of contact-angle measurements in Fig. 4. The contact angles of the perovskite film alone were extremely low, decreasing from $60.9^{\circ}$ to $50.4^{\circ}$ within $1 \mathrm{~min}$, and stabilizing at $36.5^{\circ}$ after $10 \mathrm{~min}$, demonstrating that the water molecules could easily penetrate into the perovskite layer. With the insertion of PQDs film on perovskite layer, a big contact angle of $100.6^{\circ}$ was measured, confirming the effective modification of the polar character of the perovskite surface [19]. More importantly, no change in contact angle within 1 min was observed and after $10 \mathrm{~min}, 99.8^{\circ}$ was fixed, apparently changing the perovskite surface from being hydrophilic to hydrophobic, thereby effectively preventing the water penetration into the perovskite layer and thus potentially improved moisture stability of the overall cell. 

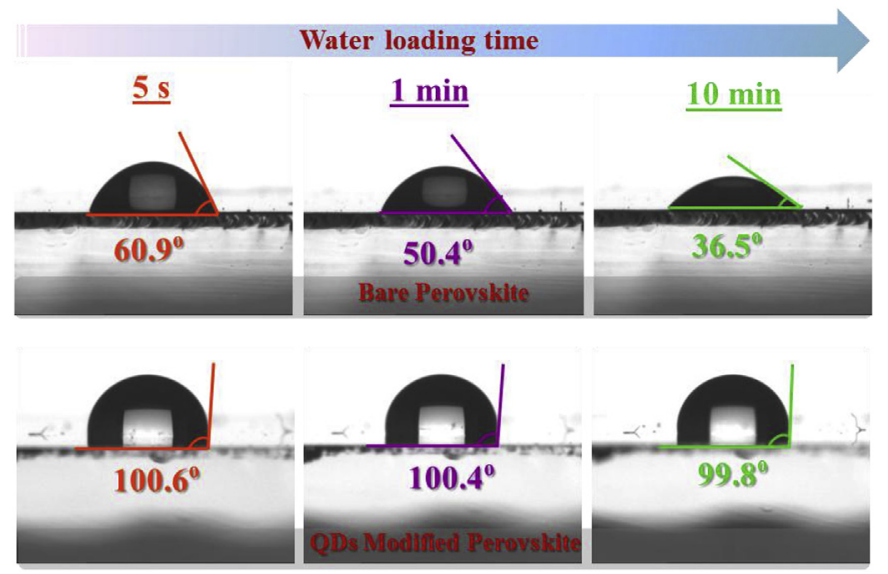

Fig. 4. Representative photographs showing the water contact angles of the bare and QDs-10 modified perovskite films. The horizontal coordinate refers to the loading time of water drops on film surfaces. Although bare perovskite readily absorbs water, its wetting ability can be considerably suppressed when functionalized with inorganic QDs layer on the surface.

Fig. $5 \mathrm{a}$ and $\mathrm{b}$ shows the images of the perovskite film surface analyzed by high-resolution scanning electron microscopy (SEM) before and after PQDs treatment, respectively, where the change in morphology is occurring at the surface of the perovskite film can be easily observed. The perovskite layers fully covered with PQDs exhibited a denser and more homogeneous surface morphology than the as-prepared film, even when a conspicuous change of the grain size is not observed. A cross-sectional SEM image of a typical n-i-p based PSCs is exemplified in Fig. $5 \mathrm{c}$ and d. When spin-coated by $10 \mathrm{mg} \mathrm{mL}^{-1}$ PQDs, a continuous, uniform, and thin ( $\sim 20 \mathrm{~nm}$ ) PQDs interlayer is formed on top of perovskite, implying close matching of PQDs surface tension with perovskite surface energy. Fig. 5 (e) and (f) show Kelvin probe force microscopy (KPFM) images and spatial maps of the local surface potential of the perovskite-QDs interface. For perovskite samples without QDs, KPFM measurements reveal an average surface potential of $0.366 \mathrm{eV}$ (Fig. 5e). When passivated by QDs on perovskite surface, KPFM measurement exhibits that the average surface potential, i.e, energy barrier, between QDs and perovskite increases to $0.571 \mathrm{eV}$ (Fig. 5f). This apparent increase in surface potential arises from the presence of a positive interfacial dipole between perovskite and QDs, and this dipole effect can cause the shift of Fermi level upward. Besides, such increased surface potential with QDs becomes more significant in preventing the back-recombination between electrons from perovskite and holes from HTM, which could improve $\mathrm{V}_{\mathrm{oc}}$ for devices passivating QDs interlayer [11,35,36].

In order to evaluate the factors that influence the photovoltaic performances of PSCs, current density-voltage (J-V) curves of these PSCs with the control and the optimal PQDs-modified perovskite films are shown in Fig. 6a, and best photovoltaic parameters as well as average are summarized in Table 1. After inserting the interfacial layer, the champion PCE increased from $19.51 \%$ to $21.14 \%$. Apparently, it is notable that this improvement on PCE resulted from the increase of $V_{o c}$ and $\mathrm{FF}$ while $\mathrm{J}_{\mathrm{sc}}$ remained almost unaltered. Such a higher $\mathrm{V}_{\mathrm{oc}}$ can be attributed to photo-induced dipole effect originating from the accumulated charge on surface of perovskite after interposed PQDs interlayer, meaning that the perovskite energy bands were positively shifted due to the increase in the dipole moment $[22,33,34]$, supported by the KPFM results in Fig. 5e and (f). The higher FF is ascribed not only enhancing hole extraction but also suppressing carrier recombination at the interfaces and boundaries in the PQDs-modified cells [12,14]. The reason for unchanging $J_{s c}$ can be interpreted that the fluorescence characteristics and light absorption properties of PQDs do not affect the light harvesting by the active layer in our experiments, as above- mentioned in absorbance measurements. To ensure the reliability of J-V measurements, the PCEs of the best-performing devices with and without PQDs were measured as a function of time, by holding a bias near the maximum power output point $(0.96 \mathrm{~V})$, as shown in Fig. $6 \mathrm{~b}$. The PCE of device with PQDs are stabilized at $20.84 \%$ over 120 s indicating that the PQDs-modified perovskite film can improve the device stability enormously. As can be seen from forward and reverse scanning $\mathrm{J}-\mathrm{V}$ curves in Fig. $6 \mathrm{c}$, while the device with PQDs-modified showed negligible hysteresis effect, hysteretic behaviors in control device could be clearly observed, confirming that the PQDs interlayer is suppressing ion migration and discarding any induced detrimental effect in the charge carrier mobility, implying well-matched charge balance $[1,37,38]$. The hysteresis effect has been also tested as a function of scanning rate of $\mathrm{J}-\mathrm{V}$ measurement. As clearly seen in Fig. S5, PQDsmodified cell has a negligible hysteresis with superior photovoltaic parameters even at higher scanning speed as compared to control cell. As can be seen in Fig. 6d, the external quantum efficiency (EQE) demonstrates values around $90 \%$ in the whole spectral range. Compared with the control device, PQDs-modified device exhibited a similar trend as EQE performance, because light harvesting is close to each other, consistent with earlier-mentioned about absorbance measurements. The integrated photocurrent densities are 23.01 (QDs-modified) and 22.87 (bare) $\mathrm{mAcm}^{-2}$, in good agreement with the $\mathrm{J}_{\mathrm{sc}}$ derived from the $\mathrm{J}-\mathrm{V}$ measurement.

The steady-state photoluminescence (PL) spectra, displayed in Fig. 6e, were recorded in order to gain insight into the charge transfer of the perovskite films with and without PQDs. Both films displayed qualitatively similar PL spectra with a maximum at around $800 \mathrm{~nm}$, and after surface modification with PQDs, perovskite film exhibits a stronger quenching. The possible explanation, resulting from quenching, could i) contain more $\mathrm{Br}$ ions leading to intrinsic lower PL yield and ii) transfer the excited electrons to the bottom perovskite [1]. When HTM was inserted on perovskite layers with and without PQDs, a slight decrease in PL spectra was observed. This observed little difference should be attributed to the carrier extraction by the HTM layer $[6,39]$. To further support this claim, additional PL measurement was fulfilled with HTM-containing configuration as shown in Fig. 6f. Consistent with above data, even with the HTM, the PL decay time were determined as 22.33 ns and $6.91 \mathrm{~ns}$ for perovskite/HTM and perovskite/PQDs/HTM, respectively, suggesting that the charge recombination was suppressed by the modified perovskite layer in complete devices. Because the PQDs interlayer at rear surface causes a quenching that can be due to the favorable electron transfer [40] in the illuminated solar cells. The interfacial layer will prevent electrons from recombining with holes at the rear surface, and therefore better device performance can be expected.

To further confirm the quenching, the time-resolved PL (TRPL) decay curves of the perovskite and PQDs-modified perovskite were analyzed (Fig. 6g). These analyses have been carried out at the PQD emission wavelength in order to study whether possible Förster Resonance Energy Transfer mediated mechanisms are involved or not. As tabulated in Table S2, the amplitude weighted lifetime of the PQDs in solution is $7.78 \mathrm{~ns}$. When prepared as a spin-coated film, the lifetime of the PQDs is shortened to $5.77 \mathrm{~ns}$ (due to possible non-radiative processes involved in the film structure). We report that modified perovskite film (consisting of PQDs on perovskite layer) exhibited faster decay time of $4.66 \mathrm{~ns}$. Since there is no HTM layer in the sample, the PL decay is attributed not to carrier extraction by the HTM layer but rather to nonradiative de-excitation. The shortened PL lifetime is due to favorable energy transfer happening at the interface with the bottom perovskite film which is attributed to FRET (Förster resonance energy transfer) mechanism in PQDs assisted PSC. PQDs layer efficiently serves as donor in energy transfer event. Thus, energy of exciton created in PQDs has been zipped to the triple-cation perovskite absorber via FRET, which improves the light harvesting of whole cell. FRET efficiency is calculated by Equation (1) as $20 \%$ from average lifetime component of 

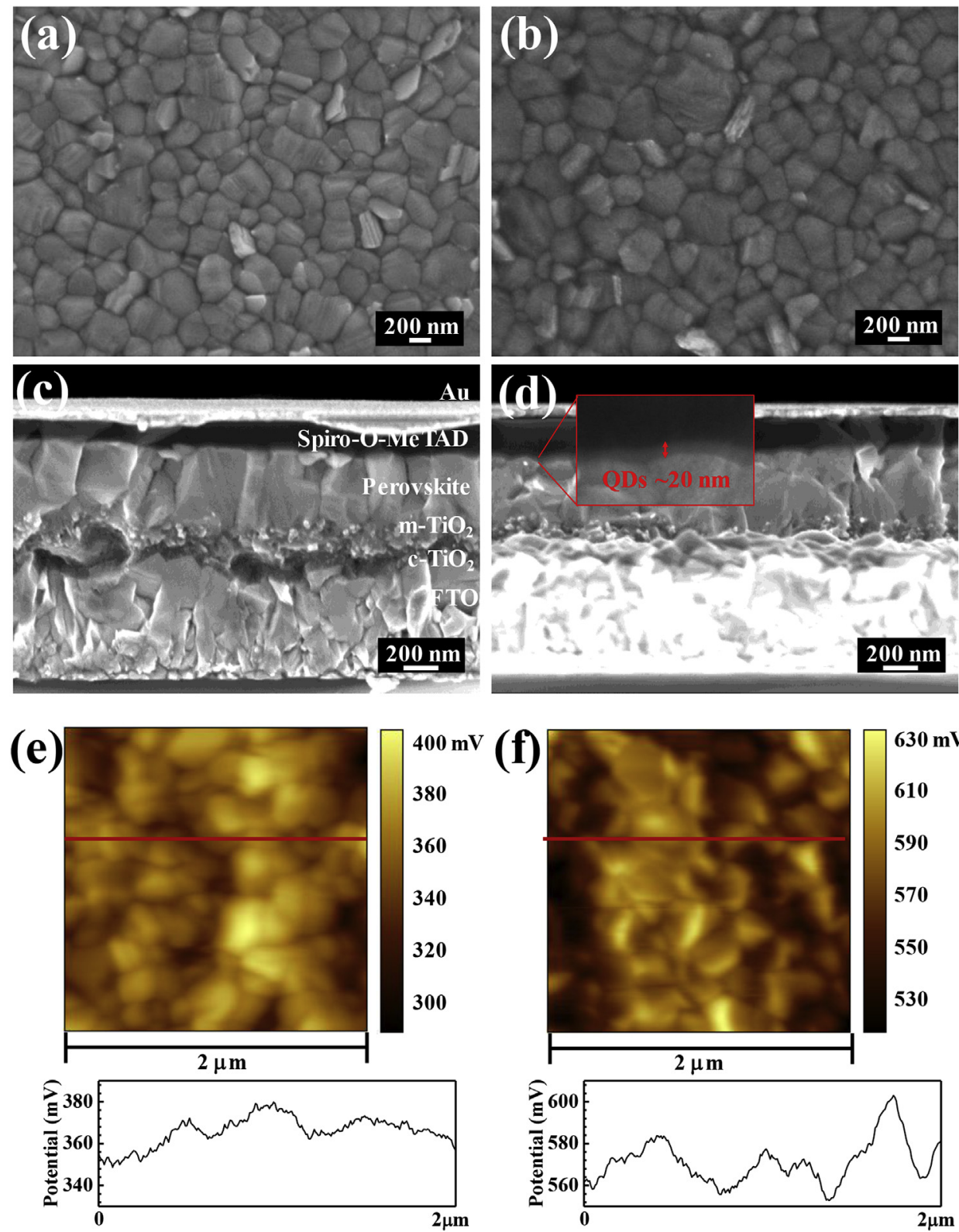

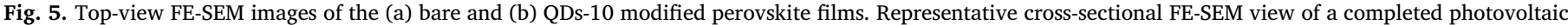

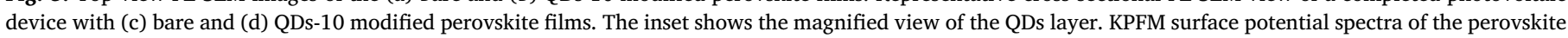
films (e) without and (f) with PQDs layer.

the only donor and donor lifetime in the presence of acceptor.

$\eta=1-\tau_{D A} / \tau_{D}$

where $\tau_{\mathrm{DA}}$ and $\tau_{\mathrm{D}}$ are donor lifetime in the presence of acceptor and only donor lifetime, taken as $4.66 \mathrm{~ns}$ and $5.77 \mathrm{~ns}$, respectively.

To examine the reproducibility of photovoltaic performance, 40 devices for each PSC structure were fabricated and evaluated in terms of photovoltaic performance (Fig. S6). The average photovoltaic performance parameters are summarized in Table 1. Besides outstanding best efficiency, the average PCEs for PQDs-modified and control cells were $20.37 \%$ and $18.79 \%$, respectively. The change trend for each average parameter is consistent with that of all parameters.

Long-term stability is an important controlling factor which accelerates the degradation of perovskite greatly under moisture ( $>40 \%$ relative humidity without any encapsulation) and continuous illumination $(>100 \mathrm{~h})$. Therefore, we investigated the both shelf and operational stability of the devices with and without PQDs as interlayer in the different ambient conditions. Firstly, the un-encapsulated cells were exposed to ambient environments with relative humidity of $40-50 \%$ at room temperature. As shown in Fig. 7a, the initial PCE of the PQDsmodified PSC remained almost unaltered, degrading only $\sim 9 \%$ of initial efficiency after 30 days, whereas the PSC of control device without PQDs rapidly dropped to $\sim 45 \%$ from its initial efficiency value in 30 days. Besides, the other photovoltaic performances including $\mathrm{V}_{\mathrm{oc}}, \mathrm{J}_{\mathrm{sc}}$, and FF for PSCs with and without PQDs have a similar tendency in terms of stability (Fig. S7). To verify the impact of the PQDs interfacial layer on the preventing degradation of PSCs, the color alteration of cells, exposed to ambient humidity-air, was observed (Fig. S8). As seen from Fig. S8, the degradation of the control devices progresses significantly within 30 days, confirming that yellow color is occurring on the top surface. For the PQDs-modified PSCs, no discernible color change was observed, indicating that the PQDs interlayer plays a significant role in shielding the perovskite structure from atmospheric moisture, thus preventing its degradation. Secondly, we also tested the 

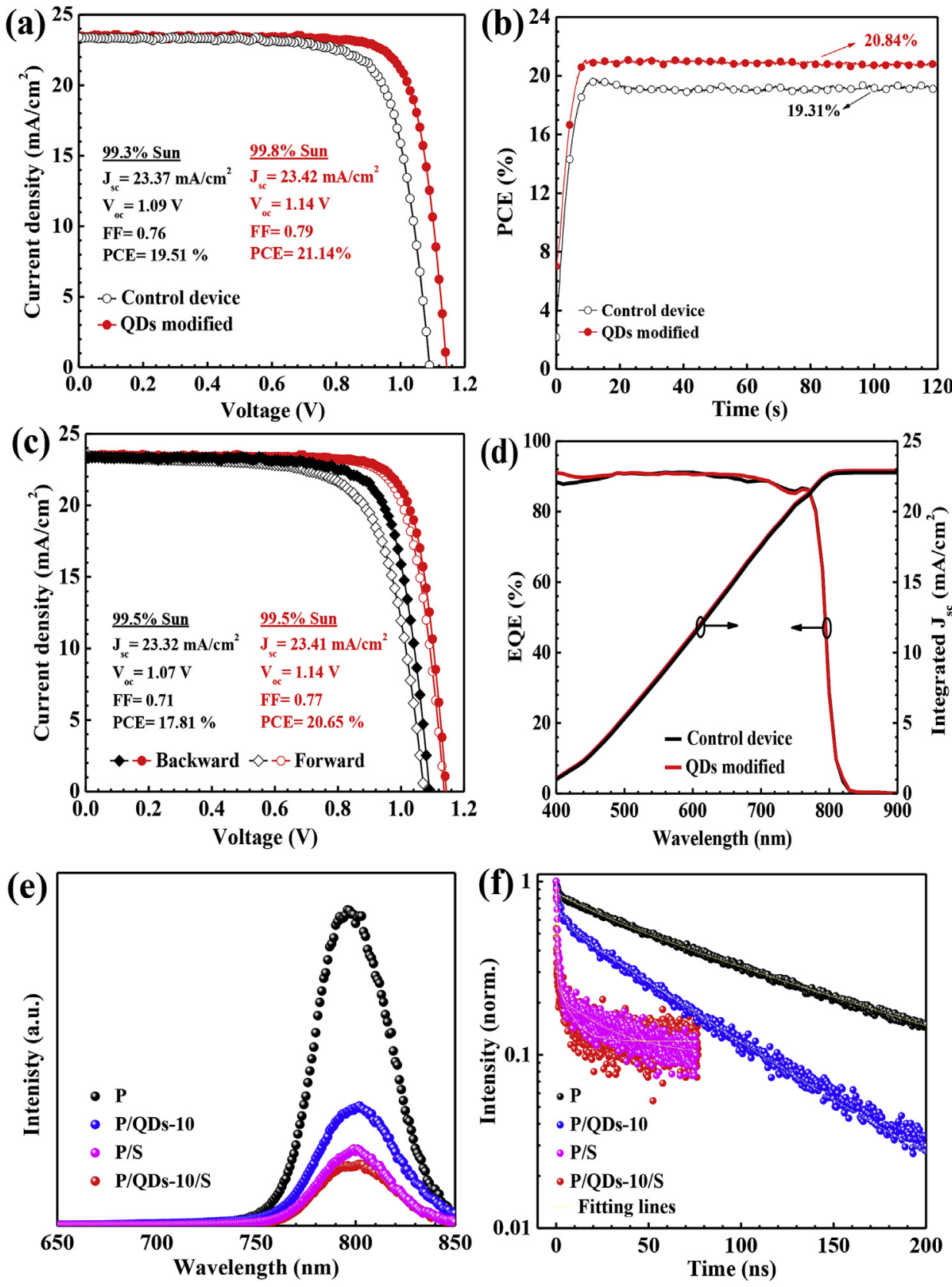

Fig. 6. (a) J-V curves for the champion cells. (b) MPPT (maximum power point tracking) for 120 s yielding a stabilized efficiency of $20.84 \%$ and $19.31 \%$ for QDs-10 modified perovskite based and control device, respectively. (c) Forward and reverse $\mathrm{J}-\mathrm{V}$ characteristics of PSCs with bare and QDs-10 modified perovskite films. The voltage scan rate for all scans was $10 \mathrm{mV} / \mathrm{s}$ and no device preconditioning, i.e., light soaking, time delay or forward voltage bias applied for a long time, was applied before starting the measurement. (d) EQE spectra of the PSCs. (e) Steady-state PL spectra (excitation at $460 \mathrm{~nm}$ ). (f) TRPL decays of the bare and QDs10 modified perovskite films with and without spiro-OMeTAD layer. All measurements reported herein were conducted with the films deposited on $\mathrm{FTO} / \mathrm{c}-\mathrm{TiO}_{2} / \mathrm{mp}-\mathrm{TiO}_{2}$. (g) TRPL decays of the PQDs in solution, bare perovskite films and QDs-10 modified perovskite films (analysis at PQD emission wavelength).

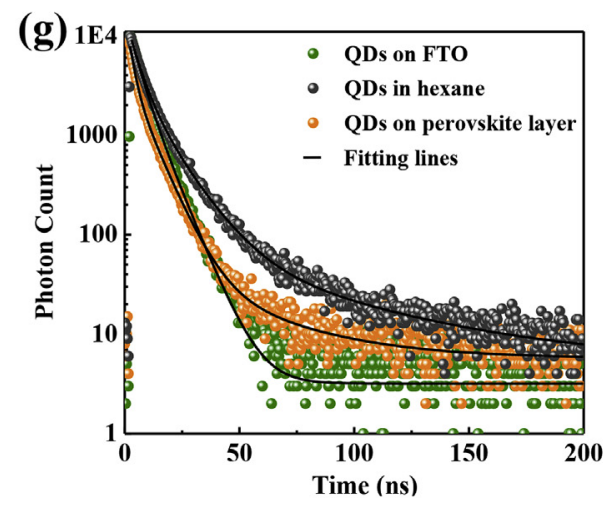


Table 1

Champion and average (40 devices) photovoltaic performances of bare and QDs-10 modified PSCs.

\begin{tabular}{|c|c|c|c|c|c|c|c|c|}
\hline \multirow[t]{2}{*}{ Devices } & \multicolumn{2}{|l|}{$\mathrm{V}_{\mathrm{oc}}(\mathrm{V})$} & \multicolumn{2}{|c|}{$\mathrm{J}_{\mathrm{sc}}\left(\mathrm{mA} \cdot \mathrm{cm}^{-2}\right)$} & \multicolumn{2}{|l|}{ FF } & \multicolumn{2}{|l|}{ PCE (\%) } \\
\hline & Champion & Average & Champion & Average & Champion & Average & Champion & Average \\
\hline Control device & 1.09 & $1.07 \pm 0.35$ & 23.37 & $23.25 \pm 0.38$ & 0.76 & $0.73 \pm 0.02$ & 19.51 & $18.79 \pm 0.50$ \\
\hline QDs modified & 1.14 & $1.10 \pm 0.23$ & 23.35 & $23.42 \pm 0.49$ & 0.79 & $0.78 \pm 0.01$ & 21.14 & $20.37 \pm 0.44$ \\
\hline
\end{tabular}
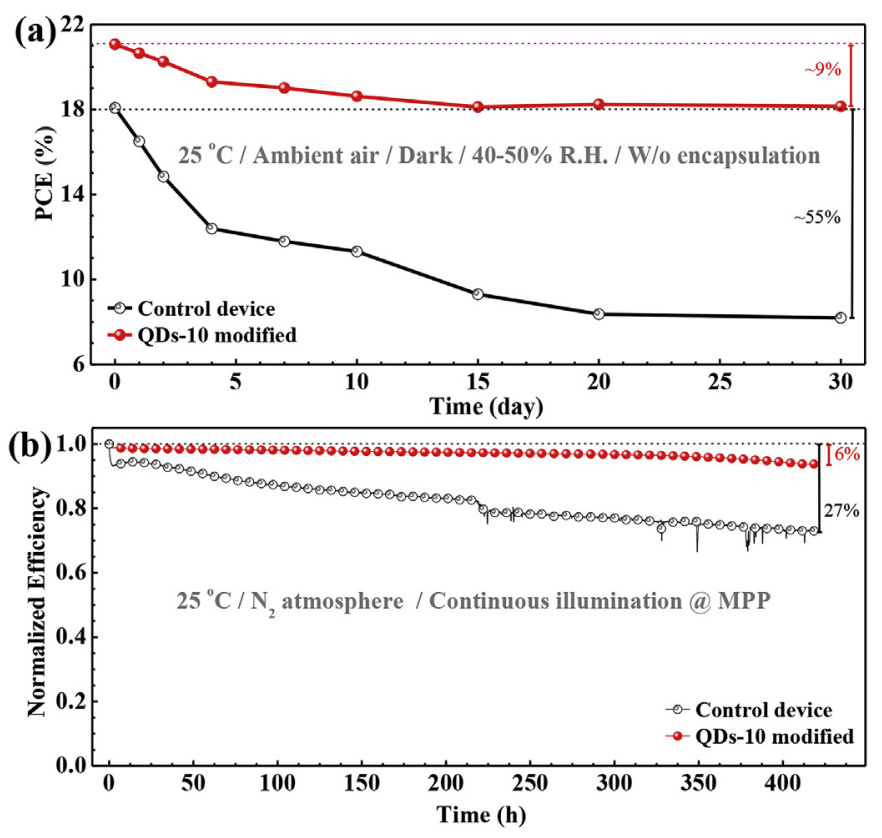

Fig. 7. The aging test for devices with the control (black line) and QDs-10 modified (red line) perovskite films, (a) The devices were measured in ambient air (at room temperature and $40-50 \%$ humidity) without any encapsulation for 30 days. (b) The devices were maintained at the maximum power point (MPP) under one sun and $\mathrm{N}_{2}$ atmosphere over $400 \mathrm{~h}$.

shelf stability of PSCs with and without PQDs-modified in a dry-air glove box without any encapsulation for 40 days (Fig. S9). As expected, the modified-cell showed a similar degradation in PCE to the control one. Thirdly, we investigate operational stability of device with and without PQDs in nitrogen atmosphere held at room temperature under continuous illumination and maximum power point tracking for $400 \mathrm{~h}$ in Fig. 7b. The PQDs-modified perovskite-based devices maintained $94 \%$ of their initial PCE over $400 \mathrm{~h}$, whereas control devices only retained $73 \%$ of their initial efficiency. The small initial drop degeneration of PQDs-modified devices could be due to ( $i$ ) the organic oleate capping agents in the PQDs, hindering the carrier transport due to longchain alkyl group, thus seriously deteriorating the device performance originating from displacement between perovskite and QDs [20,40,41] and (ii) the emergence of the partial of unreacted $\mathrm{PbI}_{2}$ presented at the surface region, which is detrimental for the long-term stability of the perovskite itself [42], and (iii) partial coverage of PQDs on perovskite film surface, thus providing insufficient protection and leading to the migration of lithium cations from spiro-OMeTAD through perovskite and even $\mathrm{TiO}_{2}[5,43,44]$. These results further illustrate that the PQDs may have a blocking/passivating effect on penetration of water and/or oxygen and/or UV-induced decomposition into the perovskite layer, which is greatly helpful to enhance the long-term device stability.

\section{Conclusions}

We developed an effective and facile approach to modify the perovskite/HTM interface by utilizing $\mathrm{CsPbBr}_{1.85} \mathrm{I}_{1.15}$ PQDs interfacial layer, and demonstrated highly stable and efficient PSCs, simultaneously, at moisture and light-soaking conditions. By employing this approach, we have achieved the best efficiency of $21.14 \%$ with a solid increase in $\mathrm{FF}$ and $\mathrm{V}_{\mathrm{oc}}$. This enhancement was further confirmed by Förster Resonance Energy Transfer mediated mechanisms on the basis of favorable energy transfer occuring at the interface. More importantly, both the efficiency of the un-encapsulated cells retained more than $90 \%$ of their initial level even after 30 days in a high humidity environment, and these cells maintained $94 \%$ of their initial efficiency value over $400 \mathrm{~h}$ under continuous illumination at $\mathrm{N}_{2}$ atmosphere, clearly demonstrating their tolerance to humidity and light, thus exhibiting superior long-term stability. The PQDs interlayer plays a few important roles: (i) It pulls holes from inner perovskite layers to the surface (HTL/perovskite interface) by creating interfacial dipoles, enhancing hole transport ability, (ii) interfacial dipoles prevent backflow of electrons from CB of perovskite to HTM direction and suppress the charge recombination, and thereby built-in potential increase, (iii) shielding the perovskite structure from atmospheric moisture and light exposure as well as ion migration, causing its degradation, and thus improving stability of cells. Accordingly, this work suggests an effective approach that demonstrating the promising potential of inorganic PQDs based materials as an interfacial layer for realizing high-performance and stable PSCs in future.

\section{Conflicts of interest}

There are no conflicts to declare.

\section{Acknowledgements}

EM acknowledges TUBA GEBIP award. EM and YA acknowledge Abdullah Gul University Scientific Research Project no: FAB-2015-10.

\section{Appendix A. Supplementary data}

Supplementary data related to this article can be found at https:// doi.org/10.1016/j.nanoen.2019.03.091.

\section{References}

[1] K.T. Cho, S. Paek, G. Grancini, C. Roldán-Carmona, P. Gao, Y. Lee, M.K. Nazeeruddin, Highly efficient perovskite solar cells with a compositionally engineered perovskite/hole transporting material interface, Energy Environ. Sci. 10 (2017) 621-627.

[2] W.S. Yang, J.H. Noh, N.J. Jeon, Y.C. Kim, S. Ryu, J. Seo, S.I. Seok, High-performance photovoltaic perovskite layers fabricated through intramolecular exchange, Science 348 (2015) 1234-1237.

[3] S.D. Stranks, S.D. Stranks, G.E. Eperon, G. Grancini, C. Menelaou, M.J.P. Alcocer, T. Leijtens, L.M. Herz, A. Petrozza, H.J. Snaith, Electron-hole diffusion lengths exceeding, Science 342 (2014) 341-344.

[4] M.I. Saidaminov, A.L. Abdelhady, B. Murali, E. Alarousu, V.M. Burlakov, W. Peng, I. Dursun, L. Wang, Y. He, G. MacUlan, A. Goriely, T. Wu, O.F. Mohammed, O.M. Bakr, High-quality bulk hybrid perovskite single crystals within minutes by inverse temperature crystallization, Nat. Commun. 6 (2015) 1-6.

[5] J.Y. Seo, H.S. Kim, S. Akin, M. Stojanovic, E. Simon, M. Fleischer, A. Hagfeldt, S.M. Zakeeruddin, M. Grätzel, Novel p-dopant toward highly efficient and stable perovskite solar cells, Energy Environ. Sci. 11 (2018) 2985-2992.

[6] H. Zhou, Q. Chen, G. Li, S. Luo, T. -b. Song, H.-S. Duan, Z. Hong, J. You, Y. Liu, Y. Yang, Interface engineering of highly efficient perovskite solar cells, Science 345 (2014) 542-546.

[7] X. Li, M.I. Dar, C. Yi, J. Luo, M. Tschumi, S.M. Zakeeruddin, M.K. Nazeeruddin, 
H. Han, M. Grätzel, Improved performance and stability of perovskite solar cells by crystal crosslinking with alkylphosphonic acid $\omega$-ammonium chlorides, Nat. Chem. 7 (2015) 703-711.

[8] J.H. Noh, S.H. Im, J.H. Heo, T.N. Mandal, S. Il Seok, Chemical management for colorful, efficient, and stable inorganic-organic hybrid nanostructured solar cells, Nano Lett. 13 (2013) 1764-1769.

[9] M. Saliba, T. Matsui, J.Y. Seo, K. Domanski, J.P. Correa-Baena, M.K. Nazeeruddin, S.M. Zakeeruddin, W. Tress, A. Abate, A. Hagfeldt, M. Grätzel, Cesium-containing triple cation perovskite solar cells: improved stability, reproducibility and high efficiency, Energy Environ. Sci. 9 (2016) 1989-1997.

[10] M. Cha, P. Da, J. Wang, W. Wang, Z. Chen, F. Xiu, G. Zheng, Z.-S. Wang, Enhancing perovskite solar cell performance by interface engineering using $\mathrm{CH}_{3} \mathrm{NH}_{3} \mathrm{PbBr} 0.9$ I $_{21}$ quantum dots, J. Am. Chem. Soc. 138 (2016) 8581-8587.

[11] H. Zai, C. Zhu, H. Xie, Y. Zhao, C. Shi, Z. Chen, X. Ke, M. Sui, C. Chen, J. Hu, Q. Zhang, Y. Gao, H. Zhou, Y. Li, Q. Chen, Congeneric incorporation of $\mathrm{CsPbBr}_{3}$ nanocrystals in a hybrid perovskite heterojunction for photovoltaic efficiency enhancement, ACS Energy Lett. 3 (2018) 30-38.

[12] X. Zhang, Q. Wang, Z. Jin, Y. Chen, H. Liu, J. Wang, Y. Li, S.F. Liu, Graphdiyne quantum dots for much improved stability and efficiency of perovskite solar cells, Adv. Mater. Interfaces. 5 (2018) 1-11.

[13] A. Capasso, F. Matteocci, L. Najafi, M. Prato, J. Buha, L. Cina, V. Pellegrini, A. Di Carlo, F. Bonaccorso, Few-layer $\mathrm{MoS}_{2}$ flakes as active buffer layer for stable perovskite solar cells, Adv. Energy Mater. 6 (2016) 1-12.

[14] P.Y. Su, L.B. Huang, J.M. Liu, Y.F. Chen, L.M. Xiao, D. Bin Kuang, M. Mayor, C.Y. Su, A multifunctional poly-N-vinylcarbazole interlayer in perovskite solar cells for high stability and efficiency: a test with new triazatruxene-based hole transporting materials, J. Mater. Chem. A. 5 (2017) 1913-1918.

[15] J.A. Christians, R.C.M. Fung, P.V. Kamat, An inorganic hole conductor for organolead halide perovskite solar cells improved hole conductivity with copper iodide, J. Am. Chem. Soc. 136 (2014) 758-764.

[16] J. Seo, S. Park, Y. Chan Kim, N.J. Jeon, J.H. Noh, S.C. Yoon, S. Il Seok, Benefits of very thin PCBM and LiF layers for solution-processed p-i-n perovskite solar cells, Energy Environ. Sci. 7 (2014) 2642-2646.

[17] Y.S. Kwon, J. Lim, H.J. Yun, Y.H. Kim, T. Park, A diketopyrrolopyrrole-containing hole transporting conjugated polymer for use in efficient stable organic-inorganic hybrid solar cells based on a perovskite, Energy Environ. Sci. 7 (2014) 1454-1460.

[18] S. Akin, Y. Liu, M.I. Dar, S.M. Zakeeruddin, M. Grätzel, S. Turan, S. Sonmezoglu, Hydrothermally processed $\mathrm{CuCrO}_{2}$ nanoparticles as an inorganic hole transporting material for low-cost perovskite solar cells with superior stability, J. Mater. Chem. A. 6 (2018) 20327-20337.

[19] S. Vidal, M. Izquierdo, S. Filippone, I. Fernández, S. Akin, J.-Y. Seo, S.M. Zakeeruddin, M. Grätzel, N. Martín, Site-selective synthesis of $\beta$-[70]PCBMlike fullerenes: efficient application in perovskite solar cells, Chem. Eur J. 25 (2019) 3224.

[20] J.W. Xiao, S. Ma, S. Yu, C. Zhou, P. Liu, Y. Chen, H. Zhou, Y. Li, Q. Chen, Ligand engineering on CdTe quantum dots in perovskite solar cells for suppressed hysteresis, Nano Energy 46 (2018) 45-53.

[21] L. Hu, W. Wang, H. Liu, J. Peng, H. Cao, G. Shao, Z. Xia, W. Ma, J. Tang, PbS colloidal quantum dots as an effective hole transporter for planar heterojunction perovskite solar cells, J. Mater. Chem. A. 3 (2015) 516-518.

[22] S. Shukla, S. Shukla, L.J. Haur, S.S.H. Dintakurti, G. Han, A. Priyadarshi, T. Baikie, S.G. Haisalkar, N. Mathews, Effect of formamidinium/cesium substitution and $\mathrm{PbI}_{2}$ on the long-term stability of triple-cation perovskites, ChemSusChem 10 (2017) 3804-3809.

[23] R.J. Sutton, G.E. Eperon, L. Miranda, E.S. Parrott, B.A. Kamino, J.B. Patel, M.T. Hörantner, M.B. Johnston, A.A. Haghighirad, D.T. Moore, H.J. Snaith, Bandgap-tunable cesium lead halide perovskites with high thermal stability for efficient solar cells, Adv. Energy Mater. 6 (2016) 1-6.

[24] X. Li, Y. Wu, S. Zhang, B. Cai, Y. Gu, J. Song, H. Zeng, CsPbX3 quantum dots for lighting and displays: room-temperature synthesis, photoluminescence superiorities, underlying origins and white light-emitting diodes, Adv. Funct. Mater. 26 (2016) 2435-2445.

[25] J. Song, J. Li, X. Li, L. Xu, Y. Dong, H. Zeng, Quantum dot light-emitting diodes based on inorganic perovskite cesium lead halides $\left(\mathrm{CsPbX}_{3}\right)$, Adv. Mater. 27 (2015) $7162-7167$.

[26] S. Sidhik, D. Esparza, A. Martínez-Benítez, T. Lopez-Luke, R. Carriles, I. Mora-Sero, E. de la Rosa, Enhanced photovoltaic performance of mesoscopic perovskite solar cells by controlling the interaction between $\mathrm{CH}_{3} \mathrm{NH}_{3} \mathrm{PbI}_{3}$ films and $\mathrm{CsPbX}$ perovskite nanoparticles, J. Phys. Chem. C 121 (2017) 4239-4245.

[27] Y. Chen, L. Li, Z. Liu, N. Zhou, Q. Chen, H. Zhou, Photon management for efficient hybrid perovskite solar cells via synergetic localized grating and enhanced fluorescence effect, Nano Energy 40 (2017) 540-549.

[28] X. Di, L. Shen, J. Jiang, M. He, Y. Cheng, L. Zhou, X. Liang, W. Xiang, Efficient white LEDs with bright green-emitting $\mathrm{CsPbBr}_{3}$ perovskite nanocrystal in mesoporous silica nanoparticles, J. Alloy. Comp. 729 (2017) 526-532.

[29] B.P. Singh, S.Y. Lin, H.C. Wang, A.C. Tang, H.C. Tong, C.Y. Chen, Y.C. Lee, T.L. Tsai, R.S. Liu, Inorganic red perovskite quantum dot integrated blue chip: a promising candidate for high color-rendering in w-LEDs, RSC Adv. 6 (2016) 79410-79414.

[30] M. Salado, J. Idigoras, L. Calio, S. Kazim, M.K. Nazeeruddin, J.A. Anta, S. Ahmad, Interface play between perovskite and hole selective layer on the performance and stability of perovskite solar cells, ACS Appl. Mater. Interfaces 8 (2016) 34414-34421.

[31] M. Salado, R.K. Kokal, L. Calio, S. Kazim, M. Deepa, S. Ahmad, Identifying the charge generation dynamics in Cs +-based triple cation mixed perovskite solar cells, Phys. Chem. Chem. Phys. 19 (2017) 22905-22914.

[32] K.T. Cho, O. Trukhina, C. Roldán-Carmona, M. Ince, P. Gratia, G. Grancini, P. Gao,
T. Marszalek, W. Pisula, P.Y. Reddy, T. Torres, M.K. Nazeeruddin, Molecularly engineered phthalocyanines as hole-transporting materials in perovskite solar cells reaching power conversion efficiency of 17.5\%, Adv. Energy Mater. 7 (2017) 1601733.

[33] S. Buhbut, S. Itzhakov, I. Hod, D. Oron, A. Zaban, Photo-induced dipoles: a new method to convert photons into photovoltage in quantum dot sensitized solar cells, Nano Lett. 13 (2013) 4456-4461.

[34] J.-F. Lu, X. Lin, X. Jiao, T.R. Gengenbach, A.D. Scully, L. Jiang, B. Tan, J. Sun, B. Li, N. Pai, U. Bach, A.N. Simonov, Y.-B. Cheng, Interfacial benzenethiol modification facilitates charge transfer and improves stability of $\mathrm{cm}$-sized metal halide perovskite solar cells with up to $20 \%$ efficiency, Energy Environ. Sci. 7 (2018) 2934-2938.

[35] N. Adhikari, A. Dubey, D. Khatiwada, A.F. Mitul, Q. Wang, S. Venkatesan, A. Iefanova, J. Zai, X. Qian, M. Kumar, Q. Qiao, Interfacial study to suppress charge carrier recombination for high efficiency perovskite solar cells, ACS Appl. Mater. Interfaces 7 (2015) 26445-26454.

[36] Z. Hu, J. Miao, T. Li, M. Liu, I. Murtaza, H. Meng, Reduced interface losses in inverted perovskite solar cells by using a simple dual-functional phenanthroline derivative, Nano Energy 43 (2018) 72-80.

[37] D. Seol, A. Jeong, M.H. Han, S. Seo, T.S. Yoo, W.S. Choi, H.S. Jung, H. Shin, Y. Kim, Origin of hysteresis in $\mathrm{CH}_{3} \mathrm{NH}_{3} \mathrm{PbI}_{3}$ perovskite thin films, Adv. Funct. Mater. 27 (2017) 1701924.

[38] J.H. Heo, M.H. Lee, H.J. Han, B.R. Patil, J.S. Yu, S.H. Im, Highly efficient low temperature solution processable planar type $\mathrm{CH}_{3} \mathrm{NH}_{3} \mathrm{PbI}_{3}$ perovskite flexible solar cells, J. Mater. Chem. A. 4 (2016) 1572-1578.

[39] Y.C. Kim, N.J. Jeon, J.H. Noh, W.S. Yang, J. Seo, J.S. Yun, A. Ho-Baillie, S. Huang, M.A. Green, J. Seidel, T.K. Ahn, S. Il Seok, Beneficial effects of $\mathrm{PbI}_{2}$ incorporated in organo-lead halide perovskite solar cells, Adv. Energy Mater. 6 (2016) 1502104.

[40] Y. Shao, Z. Xiao, C. Bi, Y. Yuan, J. Huang, Origin and elimination of photocurrent hysteresis by fullerene passivation in $\mathrm{CH}_{3} \mathrm{NH}_{3} \mathrm{PbI}_{3}$ planar heterojunction solar cells, Nat. Commun. 5 (2014) 1-7.

[41] J. Li, L. Xu, T. Wang, J. Song, J. Chen, J. Xue, Y. Dong, B. Cai, Q. Shan, B. Han, H. Zeng, 50-Fold EQE improvement up to $6.27 \%$ of solution-processed all-inorganic perovskite $\mathrm{CsPbBr}{ }_{3}$ QLEDs via surface ligand density control, Adv. Mater. 29 (2017) 1603885.

[42] J. Lee, D. Kim, H. Kim, S. Seo, S.M. Cho, N. Park, Formamidinium and cesium hybridization for photo- and moisture-stable perovskite solar cell, Adv. Energy Mater. 5 (2015) 1501310.

[43] M. Saliba, T. Matsui, K. Domanski, J.-Y. Seo, A. Ummadisingu, S.M. Zakeeruddin, J.P. Correa-Baena, W.R. Tress, A. Abate, A. Hagfeldt, M. Grätzel, Incorporation of rubidium cations into perovskite solar cells improves photovoltaic performance, Science 354 (2016) 206-209.

[44] S. Paek, P. Schouwink, E.N. Athanasopoulou, K.T. Cho, G. Grancini, Y. Lee, Y. Zhang, F. Stellacci, M.K. Nazeeruddin, P. Gao, From nano- to micrometer scale: the role of antisolvent treatment on high performance perovskite solar cells, Chem. Mater. 29 (2017) 3490-3498.

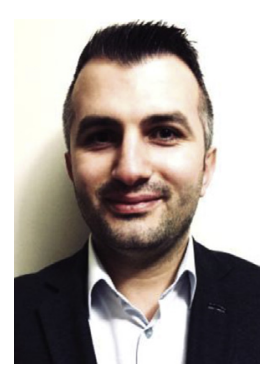

Dr. Seckin Akin pursued his $\mathrm{PhD}$ from Eskisehir Technical University (Turkey) in the area of perovskite solar cells (2019). He has been working as a Research Assistant in Karamanoglu Mehmetbey University since 2011. He was a visiting researcher at Prof. Michael Grätzel's laboratory at École Polytechnique Fédérale de Lausanne (EPFL) from 2017 to 2018 . He is involved in fundamental and applied research in the area of photovoltaics and nano-fabrication. In his research career, he has focused on a wide variety of novel materials and their photovoltaic applications in perovskite and dye-sensitized solar cells.

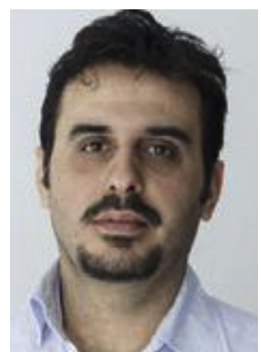

Dr. Yemliha Altintas has been working as a Research Assistant in Abdullah Gul University at Material Science and Nanotechnology Engineering Department since 2013. He received his Ph.D. degree from Abdullah Gul University Material Science and Mechanical Engineering Programme in 2018. For the last two years, he has been working as a visiting scholar at National Nanotechnology Research Center (UNAM) at Bilkent University. His current research work is focused on the synthesis of semiconductor quantum dots, nanoplatelets and metal nanoparticles and their applications in optoelectronic devices such as LEDs, colloidal lasers and solar cells. 


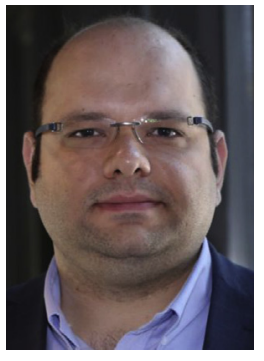

Assoc. Prof. Evren Mutlugün received the B.Sc degree in Physics from Middle East Technical University in 2005. He got his M.Sc. and Ph.D. degrees, both from Bilkent University Physics Department in 2007 and 2012 respectively. Prior to joining Abdullah Gül University as a faculty of Electrical-Electronics Engineering in 2014, he worked as a National Research Foundation Competitive Research Programme (NRF-CRP) research fellow at Nanyang Technological University, Singapore between 2012 and 2014. His research focuses on the colloidal quantum dotbased exciton harvesting systems for novel optoelectronic applications. He is the recipient of the 2017 Turkish Academy of Sciences Outstanding Young Scientist Award.

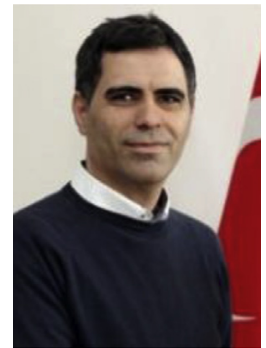

Savas SONMEZOGLU is a professor in the Department of Metallurgical and Materials Engineering at Karamanoglu Mehmetbey University; a faculty member since 2011. Also, he is the head of the Sonmezoglu Research Group since 2013. His research mainly focuses on the fabrication and design of micro/opto-electronic devices. Moreover, he has also studied the synthesis of inorganic based nano-materials. He has authored or co-authored more than 60 peerreviewed scientific publications and book chapter. 